\title{
Differential measurements of Higgs production at ATLAS and CMS
}

Toni SCULAC ${ }^{* \dagger}$

FESB, University of Split, Croatia

E-mail: toni.sculacecern.ch

Differential Higgs boson production cross sections are sensitive probes for physics beyond the Standard Model. New physics may contribute in the gluon-gluon fusion loop, the dominant Higgs boson production mechanism at the LHC, and manifest itself through deviations from the distributions predicted by the standard model. A variety of measurements are reported using the $\mathrm{H} \rightarrow \gamma \gamma$, $\mathrm{H} \rightarrow \mathrm{ZZ}^{*} \rightarrow 4 \ell$, and their combinations together with boosted $\mathrm{H} \rightarrow \mathrm{b} \overline{\mathrm{b}}$ from ATLAS and CMS Collaborations. No significant deviations from the Standard Model expectations are observed. Precision on measurements is still largely statistically limited. Finally, projections of the differential cross section measurements for the High-Luminosity LHC are reported assuming different scenarios in the extrapolation of systematical uncertainties.

7th Annual Conference on Large Hadron Collider Physics - LHCP2019

20-25 May, 2019

Puebla, Mexico

*Speaker.

${ }^{\dagger}$ on behalf of the ATLAS and CMS Collaborations 


\section{Introduction}

After the Higgs boson was discovered [1, 2, 3] by the ATLAS [4] and CMS [5] Collaborations the focus has been shifting on studying its properties. With more data collected in the LHC Run 2 it is possible to improve the precision of previous studies on the Higgs boson differential cross section $[6,7,8,9]$. Differential cross sections are measured in a fiducial phase space in order to minimize the extrapolation to the full phase space and ensure reproduciblity in calculations for future comparisons. It is crucial to test the Standard Model (SM) predictions for full spectra of observables of interest in order to probe for possible hints of the physics beyond the Standard Model (BMS). This overview discusses results from three channels considered with latest public results from the ATLAS and CMS Collaborations available at the time $[10,11,12,13,14,15,16$, $17,18,19]$.

\section{Analysis overview}

Three decay channels of the Higgs boson are used in the main differential properties studies. The $\mathrm{H} \rightarrow \gamma \gamma$ and $\mathrm{H} \rightarrow \mathrm{ZZ}^{*} \rightarrow 4 \ell$ channels provide main sensitivity and are used to extract results from both ATLAS and CMS Collaborations while the boosted $\mathrm{H} \rightarrow \mathrm{b} \overline{\mathrm{b}}$ decay channel is used in the CMS Collaboration to improve sensitivity for the Higgs transverse momentum in the high- $p_{T}$ bins. A detailed description of each analysis, together with the definition of the fiducial volume used in the differential measurements is given in respective notes and here only a very brief overview will be highlighted.

In the $\mathrm{H} \rightarrow \gamma \gamma$ analysis the signal is reconstructed by two energetic photons. Main backgrounds are from SM $\gamma \gamma, \gamma+$ jet, and di-jet processes. One of the most important aspects in the signal selection chain is the vertex assignment for photons. ATLAS uses a neural network approach that relies on track and calorimeter information for input, while CMS uses similar input information that is fed to a boosted decision tree. Additionally, CMS categorizes events according to mass resolution to further improve the sensitivity. In both cases, signal is extracted from a fit to diphoton mass spectrum. An example of the weighted di-photon spectrum is shown on the left in Fig. 1. All analysis details can be found in $[10,12]$.

In the $\mathrm{H} \rightarrow \mathrm{ZZ}^{*} \rightarrow 4 \ell$ channel the signal is fully reconstructed using four leptons with good momentum resolution. Main backgrounds come from irreducible SM contributions, $\mathrm{q} \overline{\mathrm{q}} \rightarrow \mathrm{ZZ}$ and $\mathrm{gg} \rightarrow \mathrm{ZZ}$, together with reducible $\mathrm{Z}+$ jets background that is estimated using data. This channel offers a large signal over background ratio under the Higgs peak. Events are categorized in lepton flavour to improve the sensitivity of differential cross section measurements and are extracted from fits to the four-lepton invariant mass distribution. An example of the four-lepton invariant mass distribution around the Higgs signal is shown in the middle in Fig. 1. All analysis details can be found in $[13,14]$.

To improve sensitivity in the high- $p_{T}$ bins CMS combines results with the boosted $\mathrm{H} \rightarrow \mathrm{b} \overline{\mathrm{b}}$ analysis. In this analysis boosted signal is reconstructed from a fat jet. Dominant SM backgrounds

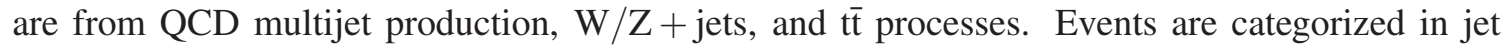
substructure and a fit to the soft-drop mass is used to extract results. An example of the soft-drop mass distribution is shown on the right in Fig. 1. All analysis details can be found in [15]. 

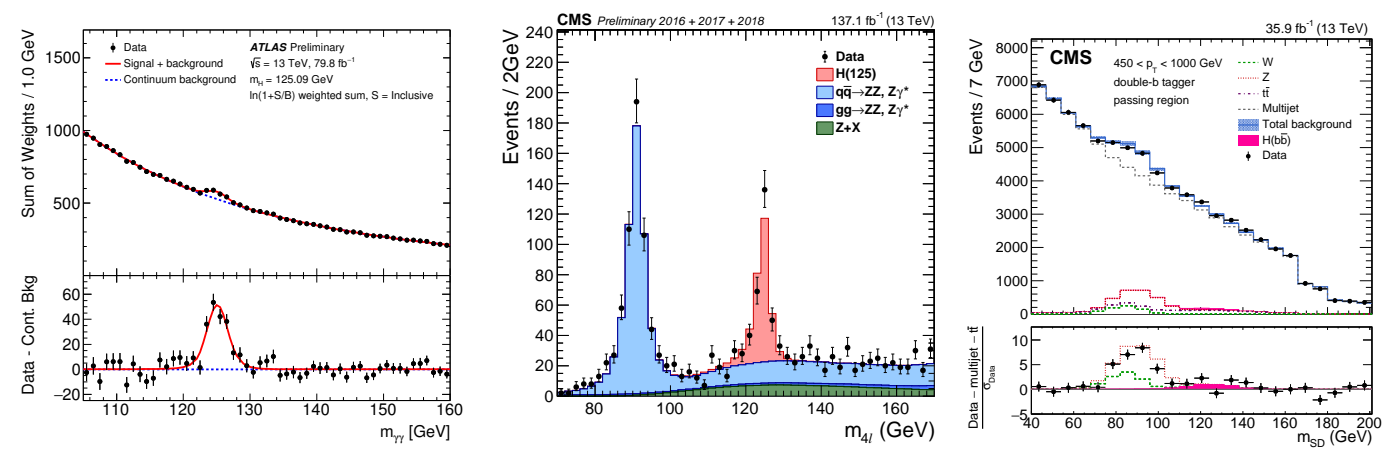

Figure 1: (Left) Weighted diphoton invariant mass spectrum in all the analysis categories observed in 79.8 $\mathrm{fb}^{-1}$ of $13 \mathrm{TeV}$ data [11]. (Middle) Distribution of the reconstructed four-lepton invariant mass $m_{4 \ell}$ with $137.1 \mathrm{fb}^{-1}$ of $13 \mathrm{TeV}$ data [14]. (Right) The soft-drop mass distributions in $35.9 \mathrm{fb}^{-1}$ of $13 \mathrm{TeV}$ data [15].

\section{Results}

A wide variety of results has been produced by both collaborations. Many variables are used to extract differential cross sections, and different data periods are covered. This section focuses only on a small fraction of these results, providing an overview of the most precise measurements on some of the key kinematic properties of the Higgs boson.

Differential measurement of the Higgs boson transverse momentum can be used to probe the perturbative QCD modeling of its production. Some of the results from two collaborations are shown in Fig. 2. Current sensitivity gives 20 to $30 \%$ precision in full range spectrum.
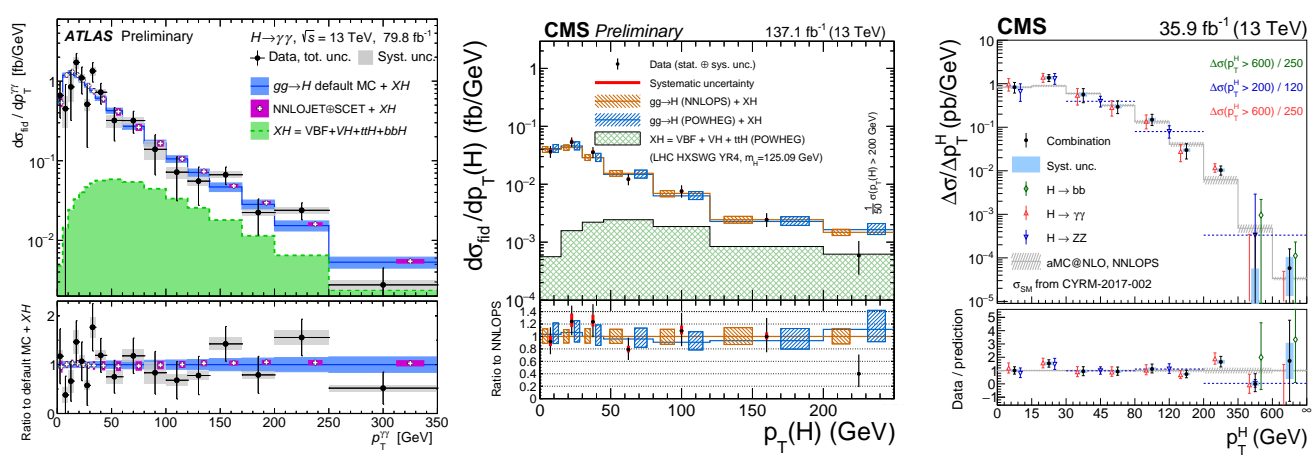

Figure 2: (Left) The fiducial differential cross sections measured in $\mathrm{H} \rightarrow \gamma \gamma$ channel as a function of $p_{T}^{\gamma \gamma}$ in $79.8 \mathrm{fb}^{-1}$ of $13 \mathrm{TeV}$ data [11]. (Middle) The results of the differential cross section measurement in the $\mathrm{H} \rightarrow \mathrm{ZZ}^{*} \rightarrow 4 \ell$ channel for $p_{T}(\mathrm{H})$ with $137.1 \mathrm{fb}^{-1}$ of $13 \mathrm{TeV}$ data [14]. (Right) Measurement of the total differential cross section as a function of $p_{T}^{\mathrm{H}}$ as a combination of three Higgs decay channels is shown in $35.9 \mathrm{fb}^{-1}$ of $13 \mathrm{TeV}$ data [17].

Variations of Higgs boson couplings would result in distortion the shape of the $p_{T}$ spectrum. In other words these measurements can be used to probe for BSM effects and reinterpret these measurements as constrains on these couplings. To summarize, no significant deviations with respect to SM expectations are observed. 
In addition to the kinematics of the Higgs boson it is also interesting to study the kinematics of additional objects in the event. For example, jet kinematics is useful to test the modeling of QCD radiation. In particular, differential measurements of the number of jets are shown in Fig. 3.
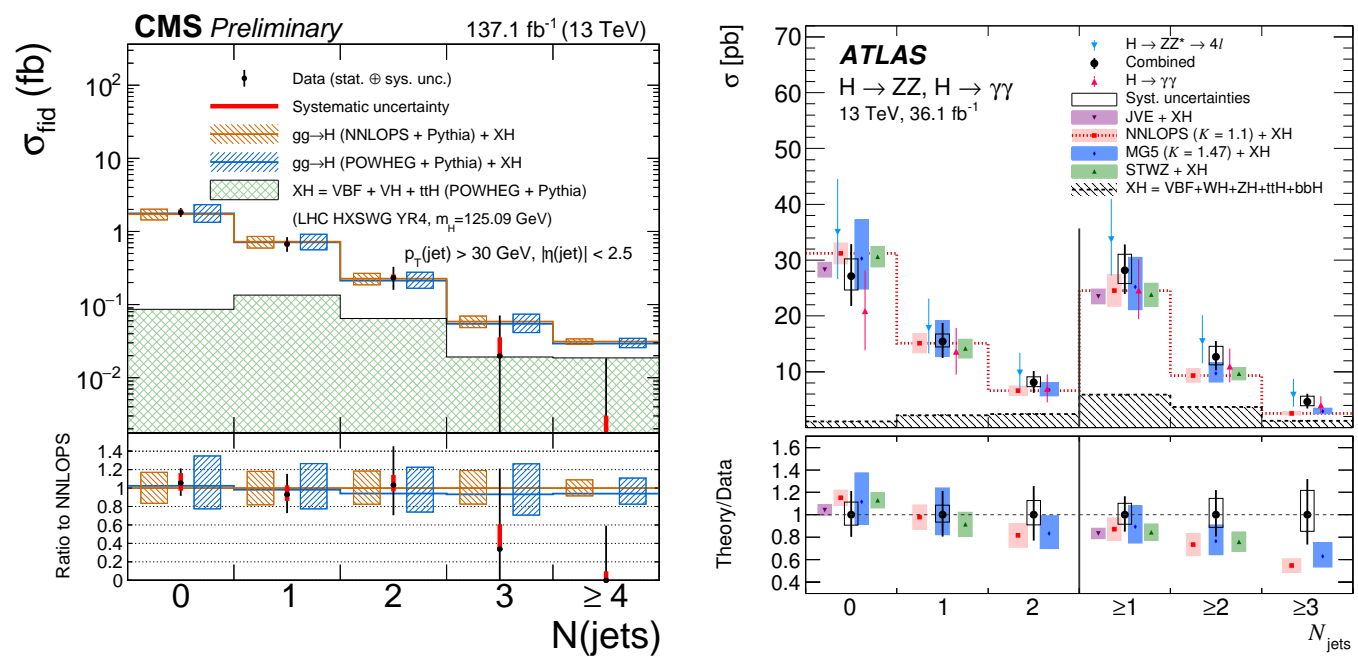

Figure 3: (Left) The results of the differential cross section measurement in the $\mathrm{H} \rightarrow \mathrm{ZZ} * 4 \ell$ channel for $N$ (jet) with $137.1 \mathrm{fb}^{-1}$ of $13 \mathrm{TeV}$ data [14]. (Right) Measurement of the total differential cross section as a function of $N$ (jet) as a combination of two Higgs decay channels is shown in $35.9 \mathrm{fb}^{-1}$ of $13 \mathrm{TeV}$ data [16].

In addition to these distributions, many other variables are studied like: Higgs boson rapidity, transeverse momentum of the leading jet, number of b-tagged jets in the event, kinematics of the sub-leading jet and the di-jet system, missing transverse momentum, and many others. Also, some double differential measurements are presented, for example $p_{T}(\mathrm{H})$ is measured in bins of $N($ jet $)$.

Finally, some projections are made by the two collaborations in order to understand the prospects of these measurements in the scope of the High-Luminosity LHC. The performance of the future detectors is assumed to be comparable to the one in Run 2 and different scenarios for the scaling of systematical uncertainties are considered. Some results are shown on Fig. 4 where one can see that the Higgs boson transverse momentum will be measured with a precision of about $5 \%$ in the low and medium region and of about $10 \%$ in the highly boosted region.

\section{Summary}

With the first part of the LHC Run 2 data analyzed by the ATLAS and CMS Collaborations, differential properties of the Higgs boson have been measured with never before achieved precision. A variety of measurements are reported by both collaborations exploiting $\mathrm{H} \rightarrow \gamma \gamma, \mathrm{H} \rightarrow \mathrm{ZZ}^{*} \rightarrow$ $4 \ell$, and $\mathrm{H} \rightarrow \mathrm{b} \overline{\mathrm{b}}$ but also combining them to achieve even higher precision. Many interesting variables have been considered for differential cross section measurements while a focus in this report has been put on the transverse momentum of the Higgs boson and the number of jets in the event. For both variables no significant deviations from the Standard Model expectations have been observed and the differential measurement of the Higgs boson transverse momentum is used 

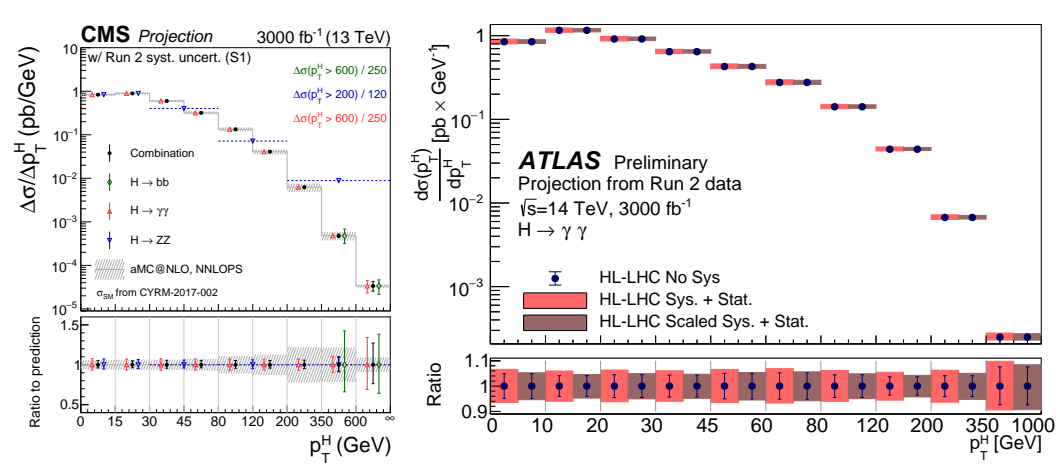

Figure 4: (Left) Projected differential cross section for the $p_{T}(\mathrm{H})$ spectrum at an integrated luminosity of $3000 \mathrm{fb}^{-1}$ [19]. (Right) Differential cross section measurement in the total phase space extrapolated to the full HL-LHC luminosity for the $p_{T}(\mathrm{H})$ spectrum [18].

to set limits on coupling modifier variations. To conclude, while extensive studies of the Higgs boson differential cross sections have been made, currently the precision of the measurements is still largely statistically limited. This is a great motivation to study the full set of data collected in Run 2 in order to improve the precision of these measurements and better understand if there are some deviations from the Standard Model expectations.

\section{Acknowledgements}

This research has been supported by the European Regional Development Fund under the grant KK.01.1.1.01.0009 (DATACROSS). 


\section{References}

[1] ATLAS Collaboration, Observation of a new particle in the search for the standard model Higgs boson with the ATLAS detector at the LHC, Phys. Lett. B 716 (2012) 1

[2] CMS Collaboration, Observation of a new boson at a mass of $125 \mathrm{GeV}$ with the CMS experiment at the LHC, Phys. Lett. B 716 (2012) 30

[3] CMS Collaboration, Observation of a new boson with mass near $125 \mathrm{GeV}$ in pp collisions at $\sqrt{s}=7$ and $8 \mathrm{TeV}$, JHEP 06 (2013) 081

[4] ATLAS Collaboration, The ATLAS experiment at the CERN Large Hadron Collider, JINST 3 S08003 (2008)

[5] CMS Collaboration, The CMS experiment at the CERN LHC, JINST 3 S08004 (2008)

[6] ATLAS Collaboration, Measurements of fiducial and differential cross sections for Higgs boson production in the diphoton decay channel at $\sqrt{s}=8 \mathrm{TeV}$ with ATLAS, JHEP 09 (2014) 112

[7] CMS Collaboration, Measurement of differential cross sections for Higgs boson production in the diphoton decay channel in pp collisions at $\sqrt{s}=8 \mathrm{TeV}$, Eur. Phys. J. C 76 (2016) 13

[8] ATLAS Collaboration, Fiducial and differential cross sections of Higgs boson production measured in the four-lepton decay channel in pp collisions at $\sqrt{s}=8 \mathrm{TeV}$ with the ATLAS detector, Phys. Lett. B 738 (2014) 234

[9] CMS Collaboration, Measurement of differential and integrated fiducial cross sections for Higgs boson production in the four-lepton decay channel in pp collisions at $\sqrt{s}=7$ and $8 \mathrm{TeV}$, JHEP 04 (2016) 005

[10] ATLAS Collaboration, Measurements of Higgs boson properties in the diphoton decay channel with $36 \mathrm{fb}^{-1}$ of pp collision data at $\sqrt{s}=13 \mathrm{TeV}$ with the ATLAS detector, Phys. Rev. D 98 (2018) 052005

[11] ATLAS Collaboration, Measurements of Higgs boson properties in the diphoton decay channel using $80 \mathrm{fb}^{-1}$ of pp collision data at $\sqrt{\mathrm{s}}=13 \mathrm{TeV}$ with the ATLAS detector, ATLAS-CONF-2018-028 (2018), http: //cdsweb.cern.ch/record/2628771

[12] CMS Collaboration, Measurement of inclusive and differential Higgs boson production cross sections in the diphoton decay channel in proton-proton collisions at $\sqrt{s}=13 \mathrm{TeV}$, JHEP 01 (2019) 183

[13] ATLAS Collaboration, Measurements of the Higgs boson production, fiducial and differential cross sections in the $4 l$ decay channel at $\sqrt{s}=13 \mathrm{TeV}$ with the ATLAS detector, ATLAS-CONF-2018-018 (2018), https://cds.cern. ch/record/2621479

[14] CMS Collaboration, Measurements of properties of the Higgs boson in the four-lepton final state in proton-proton collisions at $\sqrt{s}=13 \mathrm{TeV}$, CMS-PAS-HIG-19-001 (2019),

https://cds.cern.ch/record/2668684

[15] CMS Collaboration, Inclusive search for a highly boosted Higgs boson decaying to a bottom quark-antiquark pair, Phys. Rev. Lett. 120 (2018) 071802

[16] ATLAS Collaboration, Combined measurement of differential and total cross sections in the $\mathrm{H} \rightarrow \gamma \gamma$ and the $\mathrm{H} \rightarrow \mathrm{ZZ}^{*} \rightarrow 4 \ell$ decay channels at $\sqrt{s}=13 \mathrm{TeV}$ with the ATLAS detector, Phys. Lett. B 786 (2018) 114

[17] CMS Collaboration, Measurement and interpretation of differential cross sections for Higgs boson production at $\sqrt{s}=13 \mathrm{TeV}$, Phys. Lett. B 792 (2019) 369 
[18] ATLAS Collaboration, Prospects for differential cross-section measurements of Higgs boson production measured in decays to $\mathrm{ZZ}$ and $\gamma \gamma$ with the ATLAS experiment at the High-Luminosity LHC, ATL-PHYS-PUB-2018-040 (2018), https : / / cds . cern. ch/record/2649879/

[19] CMS Collaboration, Sensitivity projections for Higgs boson properties measurements at the HL-LHC, CMS-PAS-FTR-18-011 (2018), https : / / cds . cern. ch/record/2647699 